\title{
Applications of Laser Speckle Photography for Thermal Flow Problems
}

\author{
K. D. Kihm \\ Department of Mechanical Engineering, Texas A\&M University, College Station, \\ TX 77843-3123, USA
}

\begin{abstract}
Laser speckle photography measures projected beam deflection of a phase object. The beam deflection measured at the solid surface will directly determine the wall-temperature gradient. Thus, the laser-speckle photography allows the evaluation of the convection-heat-transfer coefficients without the need to correct for conduction and radiation heat losses, which otherwise would be necessary. Selected results and brief discussions are presented for several natural convection-heat-transfer problems. Second, high-temperature applications of laser speckle photography shows potential as a nonintrusive means of measuring flame temperature. A fairly good agreement is shown in comparison of the laser speckle data with intrusive thermocouple data. Suggestive discussions are made to further improve the accuracy and sensitivity of the laser speckle photography technique in high-temperature applications. Finally, tomographic reconstruction of laser speckle photography data is discussed using density computer-synthesized phantom fields. The conventional algebraic reconstruction technique $(A R T)$ has been modified to apply for the laser speckle tomography. The reconstruction accuracy of $A R T$ is compared with Fourier convolution $(F C)$ technique particularly under a limited number of projections. (C) 1998 Published by Elsevier Science Ltd. All rights reserved.
\end{abstract}

\section{INTRODUCTION}

Speckle photography ${ }^{1}$ refers to an optical method that measures the refractive index gradients of a phase object. It measures the amount of dislocation of optical speckles that linearly increases with an increasing refractive index gradient at the measurement point. The measured refractive index gradients are then converted into the medium temperature and density information using pertinent physical equations. Speckle photography is different from particle-image velocimetry (PIV) ${ }^{2}$ in that PIV images form as a result of Mie scattering from the physically seeded particles. Speckle photography images, 
however, are constructed by the light ray interference without the need of artificial seeding.

The basic principle of laser speckle photography has been well published in previous literature. ${ }^{3-6}$ A brief review of the operating principle is repeated here to make the paper self-contained. Consider a diffraction grating consisting of many slits, all random in shape, size, and orientation. Such a grating is possible by finely sandblasting one side of a glass plate so that the surface is randomly rough. When coherent light is incident upon this random diffraction grating, light is randomly diffracted in all directions (Fig. 1). Thus, the interference pattern viewed at a distance far from the ground glass is also a random one. That is, the random diffraction causes a region of random constructive and destructive interference. When viewed with the naked eye, this is seen as a very fine pattern of bright and dark spots, or laser speckles. When viewed at a magnification, the laser speckles are of random shapes and sizes. Analysis based on diffraction laws ${ }^{7,8}$ gives a median speckle diameter of $d=2 \cdot 44 \lambda H / D$, where $\lambda$ is the wavelength of the laser radiation, $H$ is the location of the speckle formation measured from the deflector (i.e., the groundglass surface), and $D$ is the dimension of the grain size of the ground-glass plate.

If a variable index of the refraction test field, also called a phase object, is placed in front of the ground glass, the change in the index of refraction will bend the light slightly from its original path, which previously did not encounter the test field. This additional angular refraction of light will cause the incident coherent light to strike the ground glass at a slightly different angle. It will also cause the entire random diffraction pattern to shift, or dislocate, an amount $\delta$, from its originally viewed position. A specklegram is taken photographically by overlapping the original speckles without a test field, and then with a test field. The resulting speckle photograph, called

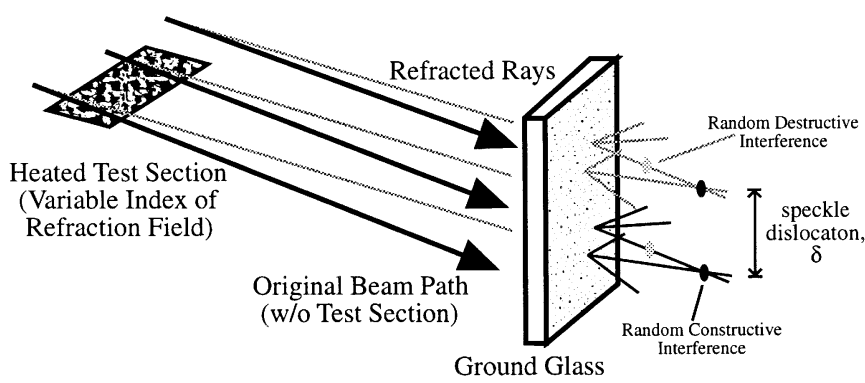

Fig. 1. Schematic illustration of formation of optical speckles and their dislocations. ${ }^{9}$ 
a specklegram, carries information on both the sizes of the speckle images and the amount of the speckle dislocations.

The optical layout of the speckle photography system closely resembles that of a Schlieren system (Fig. 2). A helium-neon laser source of $\lambda=632.8 \mathrm{~nm}$ expands to $120 \mathrm{~mm}$ in diameter, and collimates by the first parabolic mirror to form parallel light rays throughout the test section. After passing through the test section, the light rays reflect from the second parabolic mirror and collimate its focal point. After the focal point, the light rays illuminate on the optical-grade ground-glass plate whose average grain size is an order of $10 \mu \mathrm{m} .{ }^{9}$

The speckle dislocation, due to the presence of an inhomogeneous test field, is schematically illustrated in Fig. 3. The amount of speckle dislocation is given as $\delta$ on the object plane of the camera and $\Delta$ on the image plane. A geometrical similarity occurs when $\varepsilon=\alpha / m$, where the magnification of the second parabolic mirror, $m$, is equivalent to the ratio of the image distance to the object distance, i.e., $m=b / a$. The speckle dislocation $\Delta$ is

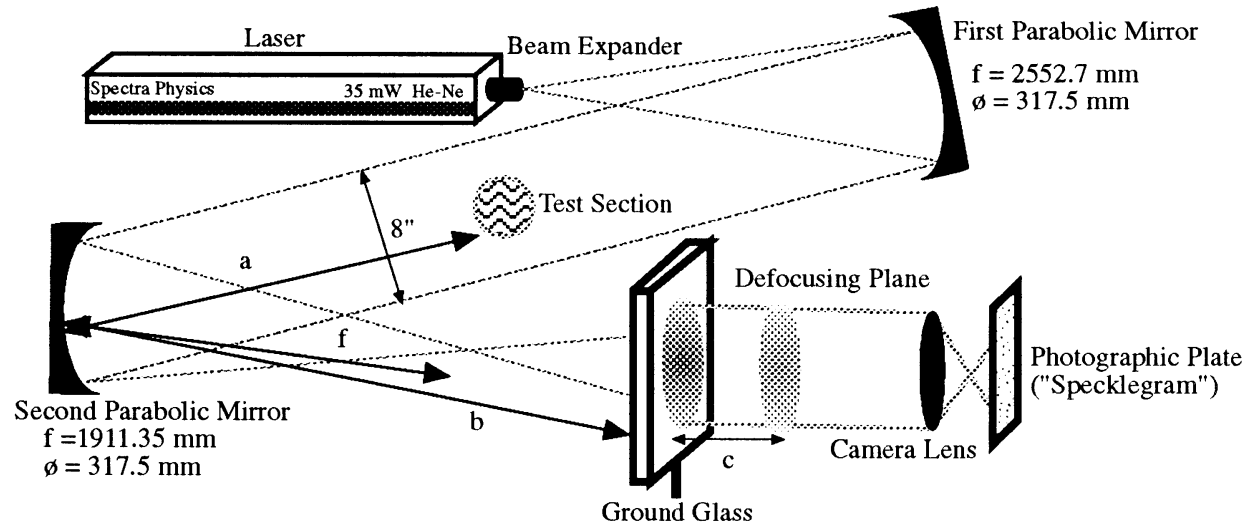

Fig. 2. Optical layout of speckle photography system. ${ }^{9}$

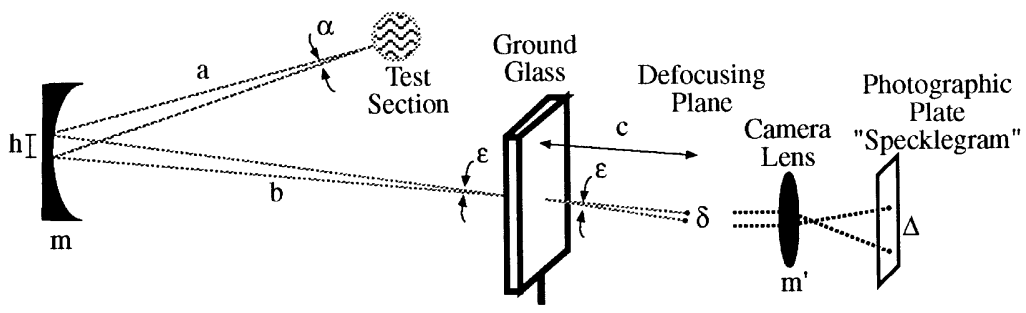

Fig. 3. Illustration of speckle dislocations by ray optics. ${ }^{9}$ 
then expressed as

$$
\Delta=m^{\prime} \times \delta=m^{\prime} \times c \times \varepsilon=\frac{m^{\prime}}{m} \times c \times \alpha
$$

where $m^{\prime}$ is the magnification of the camera lens. The 'defocusing distance' $c$ represents the distance between the ground glass and the focal plane of the camera lens.

The test section image is focused onto the ground glass. However, the camera-focusing plane for the speckle image recording must be slightly away from the ground glass since the speckle dislocations are not detectable on the ground glass itself. Defocusing is thus inevitable to record a detectable dislocation of the speckle images. On the other hand, the defocusing distance must be minimized to reduce blurring the recorded image. Estimating the optimal defocusing distance must consider both optical parameters, test conditions, and hands-on experience. In addition, the analysis for calculating the image blur ${ }^{10}$ suggests that the test object be placed near the optical axis to reduce the blur, and that mirrors of larger diameters with longer focal lengths will enhance the measurement sensitivity of the speckle photography system.

The point-by-point interrogation ${ }^{11}$ of a specklegram determines the distance of the speckle dislocation $\Delta$ from the spacing measurement of Young's fringes, ${ }^{12}$ which are generated by illuminating the specklegram with a lowpower laser (Fig. 4). The fringe spacing, $s$, decreases with an increase of the speckle dislocation $\Delta$, i.e.,

$$
s=\left(s_{y}, s_{z}\right)=\frac{\lambda \times d}{\Delta}
$$

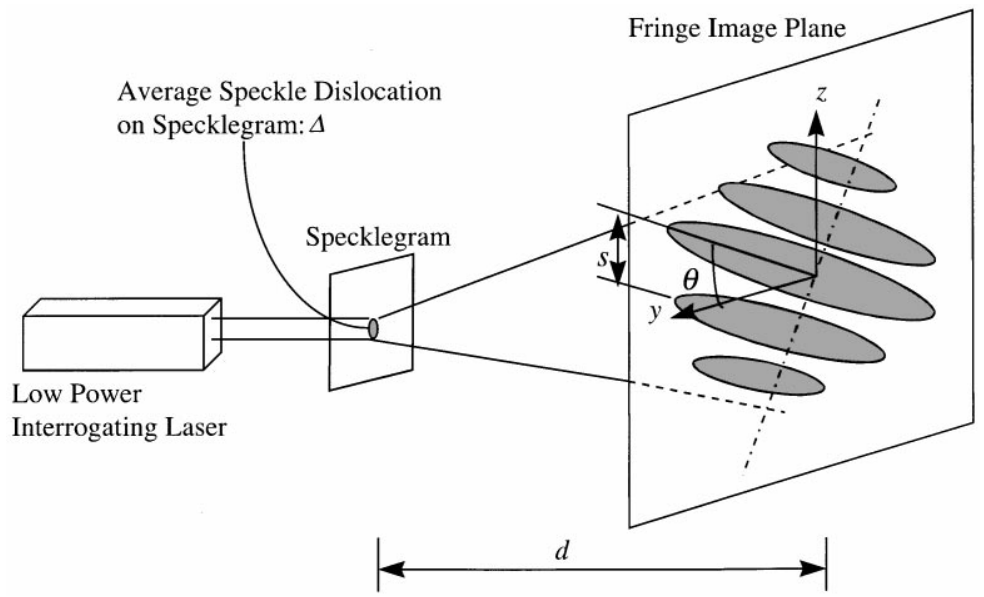

Fig. 4. Point-by-point data analysis by measuring Young's fringes generated from specklegram. 
where $s_{y}=s \cos \theta$ and $s_{z}=s \sin \theta$, with $\theta$ being the tilting angle of the fringe orientation on the $y-z$ imaging plane. $\lambda$ denotes the wavelength of the interrogating laser beam ( $\lambda=632.8 \mathrm{~nm}$ for a helium-neon laser) and $d$ is the distance between the specklegram and the imaging screen.

Within the context of geometrical optics, Fermat's principle on the propagation of a single light ray through a medium states that the light wave will take a path that involves the least travel time. The use of a paraxial approximation, i.e., the amount of ray diffraction in the medium being very small and the light ray remaining nearly in a straight fashion (Fig. 5), the diffraction angle components, $\varepsilon_{y}$ and $\varepsilon_{z}$, are expressed as the path integral along the optical axis: ${ }^{13}$

$$
\begin{aligned}
\tan \varepsilon_{y} \cong \varepsilon_{y}\left(y, z=z_{k}\right) & =\int_{\zeta_{1}}^{\zeta_{2}} \frac{1}{n(x, y)} \frac{\partial n(x, y)}{\partial y} \mathrm{~d} x \\
\tan \varepsilon_{z} \cong \varepsilon_{z}\left(y, z=z_{k}\right) & =\int_{\zeta_{1}}^{\zeta_{2}} \frac{1}{n(x, y)} \frac{\partial n(x, y)}{\partial z} \mathrm{~d} x
\end{aligned}
$$

Combining eqns (1), (2), (3a) and (3b) constitutes the basic relations between the measured fringe spacing and the medium refractive index field as

$$
\begin{aligned}
& s_{y}\left(y, z_{k}\right)=\frac{m}{m^{\prime}} \frac{\lambda d}{c}\left[\int_{\zeta_{1}}^{\zeta_{2}} \frac{1}{n} \frac{\partial n}{\partial y} \mathrm{~d} x\right]^{-1} \\
& s_{z}\left(y, z_{k}\right)=\frac{m}{m^{\prime}} \frac{\lambda d}{c}\left[\int_{\zeta_{1}}^{\zeta_{2}} \frac{\partial}{n} \frac{\partial n}{\partial z} \mathrm{~d} x\right]^{-1}
\end{aligned}
$$

where the fringe spacing ' $s$ ' is inversely proportional to the line-of-sight integral of the refractive index gradient along the occupied laser ray.

The primary advantages of speckle photography over other optical methods relying on ray refraction, such as wavefront interferometry, Schlieren

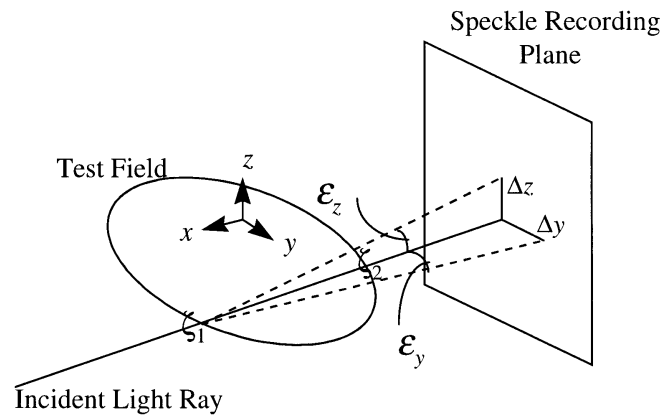

Fig. 5. Ray diffraction due to a phase object and their deflection angles. 
method, and holography, can be summarized as ${ }^{14}$ (i) the optical setup of speckle photography is simple because the single-beam path needs no reference beam, (ii) its requirements concerning mechanical stability against the external disturbance are less stringent, and (iii) specklegrams have higher spatial resolution of information density than other methods.

\section{MEASUREMENT OF NATURAL CONVECTION HEAT TRANSFER COEFFICIENTS}

For a simple geometry, such as a vertical isothermal wall, eqn (4b) is negligible since the gradient in the $z$-direction is small compared to the gradient in the $y$-direction. The refractive index gradient in the $y$-direction is assumed to remain constant along the ray direction $(x)$. Thus, eqn (4a) reduces to

$$
\frac{\partial n}{\partial y}=\frac{m}{m^{\prime}} \frac{\lambda d}{c L} \frac{1}{s_{y}}
$$

where the fringe spacing, $s_{y}$, becomes narrower for larger refractive index gradients, and the spacing becomes infinite as the gradient diminishes. Equation (5) is also expressed as,

$$
\frac{\partial T}{\partial y}=\frac{m}{m^{\prime}} \frac{\lambda d}{c L} \frac{1}{s_{y}}\left(\frac{\partial n}{\partial T}\right)^{-1}
$$

where $L$ denotes the dimension of the test field measured along the optical ray. $n$ is assumed approximately unity, and the refractive index change per unit temperature, $\partial n / \partial T$, for air at $\lambda=632.8 \mathrm{~nm}$ wavelength of a helium-neon laser, is given as ${ }^{15}$

$$
\frac{\partial n}{\partial T}=-\frac{1 \cdot 075 \times 10^{-6}}{(1+0.00368184 T)^{2}}
$$

where $T$ is in ${ }^{\circ} \mathrm{C}$. The use of the ideal gas equation of state, together with the Gladstone-Dale relationship, ${ }^{16} n=1+K \rho$ with $K$ being the GladstoneDale constant (for air $K=0.2257 \times 10^{-3} \mathrm{~m}^{3} / \mathrm{kg}$ at $\lambda=632.8 \mathrm{~nm}$ ), converts eqn (5) into an expression for the density gradient, i.e.,

$$
\frac{\partial \rho}{\partial y}=\frac{m}{m^{\prime}} \frac{\lambda d}{c L} \frac{1}{s_{y}} \frac{1}{K}
$$

A small fringe spacing reflects a large temperature and density gradients [Eqns (6) and (8), respectively]. Note that the speckle dislocates in the direction of temperature gradient, and fringes form in layers perpendicular to the average direction of the speckle dislocations. 
The heat transfer to or from a solid surface can simulate many popular engineering problems, for example, cooling in electronic packaging, turbineblade cooling, and a variety of heat exchangers. Assuming a steady problem with uniformly distributed heat sources, the energy-flux balance yields $\dot{q}_{\text {total }}^{\prime \prime}=$ $\dot{q}_{\text {radiation }}^{\prime \prime}+\dot{q}_{\text {conduction }}^{\prime \prime}+\dot{q}_{\text {convection. }}^{\prime \prime}$ The traditional experiment involves setting the total heat generation from the surface heater, $\dot{q}_{\text {total }}^{\prime \prime}$, estimating the heat radiation, $\dot{q}_{\text {radiation }}^{\prime \prime}$, and the conduction losses, $\dot{q}_{\text {conduction. }}^{\prime \prime}$ After substracting the latter two terms from the total heat flux, the convection-heat-transfer coefficient $h$ is determined, i.e.,

$$
h=\frac{\dot{q}_{\text {convection }}^{\prime \prime} \equiv \dot{q}_{\text {total }}^{\prime \prime}-\dot{q}_{\text {radiation }}^{\prime \prime}-\dot{q}_{\text {conduction }}^{\prime \prime}}{T_{\mathrm{w}}-T_{\infty}}
$$

This method requires that the whole experimental apparatus, other than the test surface, be wrapped with bulky insulation material to reduce the losses to the environment. The uncertainties in determining the radiation heat losses are as equally difficult to quantify as the conduction losses. An incorrect evaluation of these heat losses can significantly affect the measurement accuracy and uncertainties, especially for natural convection problems where the conduction/radiation heat loss amounts can be as significant as the amount of convection-heat transfer itself.

Such difficulties are alleviated if the heat-transfer coefficient, $h$, is directly measured without involving the total heat generation rate. The laser speckle photography technique directly measures $h$, which provides reduced uncertainties and improved measurement resolution, thereby enhanced data accuracy. ${ }^{17}$ The flow velocity in the region very close to the wall is practically zero and the heat transfer into the fluid takes place only by radiation and conduction. The heat transfer in the region away from the wall replaces the conduction with a convection mode while maintaining the radiation constant. By equating the two heat-transfer patterns with Newton's law of cooling, ${ }^{18}$ the convection-heat-transfer coefficient is given as

$$
h=\frac{-k(\partial T / \partial y)_{\mathrm{wall}}}{T_{\mathrm{w}}-T_{\infty}}
$$

where the $y$-coordinate is normal to the heater surface. The wall-temperature gradient $(\partial T / \partial y)_{\text {wall }}$ is measured from the analysis of fringe spacing [eqn (6)]. The wall temperature and ambient temperature are measured by traditional means such as a thermocouple probe.

The vertical heated plate has been the standard for comparing different optical methods, particularly because of its well-established analytical/numerical solutions. ${ }^{19-23}$ Figure 6 shows the fringe patterns at five locations inside and outside of the thermal layer of an isothermal vertical wall. ${ }^{24}$ The steep 


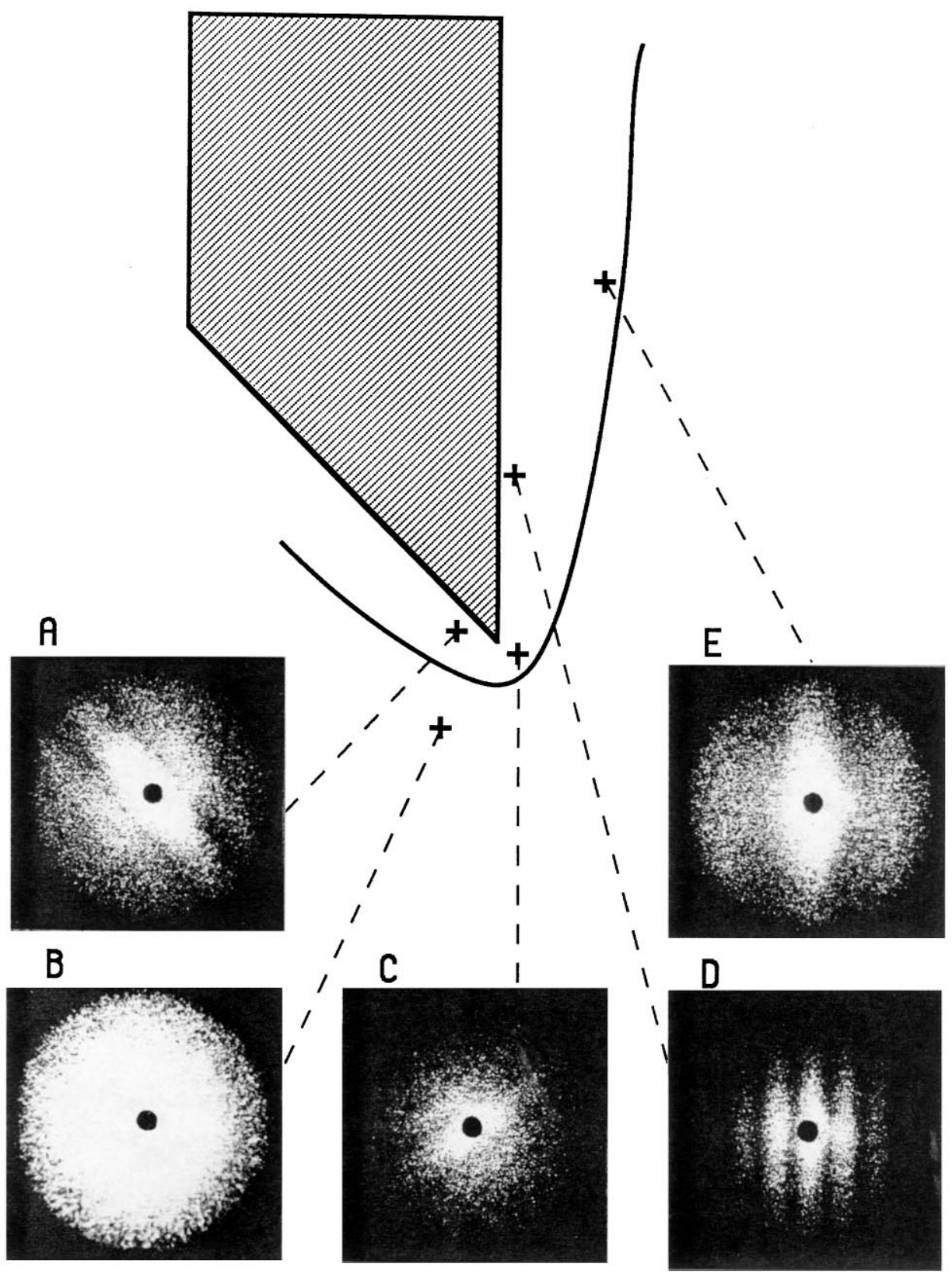

Fig. 6. Fringe spacing and orientations at five different locations around the leading edge of an isothermal vertical wall at $T_{\mathrm{w}}=86 \cdot 2^{\circ} \mathrm{C}^{24}$ 
temperature gradient near the wall gives a relatively narrower fringe spacing $(D)$, and the fringe spacing becomes wider as the edge of the boundary layer is approached $(E)$. The fringes disappear outside the thermal layer because there are no detectable dislocation of speckles $(B)$. The orientation of the fringes around the leading edge alters depending on the local temperature gradient (A and $\mathrm{C}$ ).

Numerical integration of the temperature gradient data obtained from the fringe spacing measurement provides detailed temperature profiles in the boundary layer (Fig. 7). The horizontal coordinate represents dimensionless coordinate by Grashof number, $\mathrm{Gr}_{x} \equiv g \beta\left(T_{\mathrm{w}}-T_{\infty}\right) x^{3} / v^{2}$ where the coefficient of thermal volumetric expansion $\beta \equiv 1 / T$. The similarity solution by Ostrach $^{25}$ assumed an ideal thermal boundary layer starting exactly at the leading edge. The real thermal boundary layer, however, starts before the leading edge because of the conduction effect. Therefore, the thermal boundary layer is thicker than the ideal one. ${ }^{26}$ The measured temperature distribution decreases more gradually than the theoretical profile because of the thicker boundary layer. This leading-edge effect explains why slightly higher temperature is measured compared with the theory $(x=14 \cdot 2 \mathrm{~mm})$.

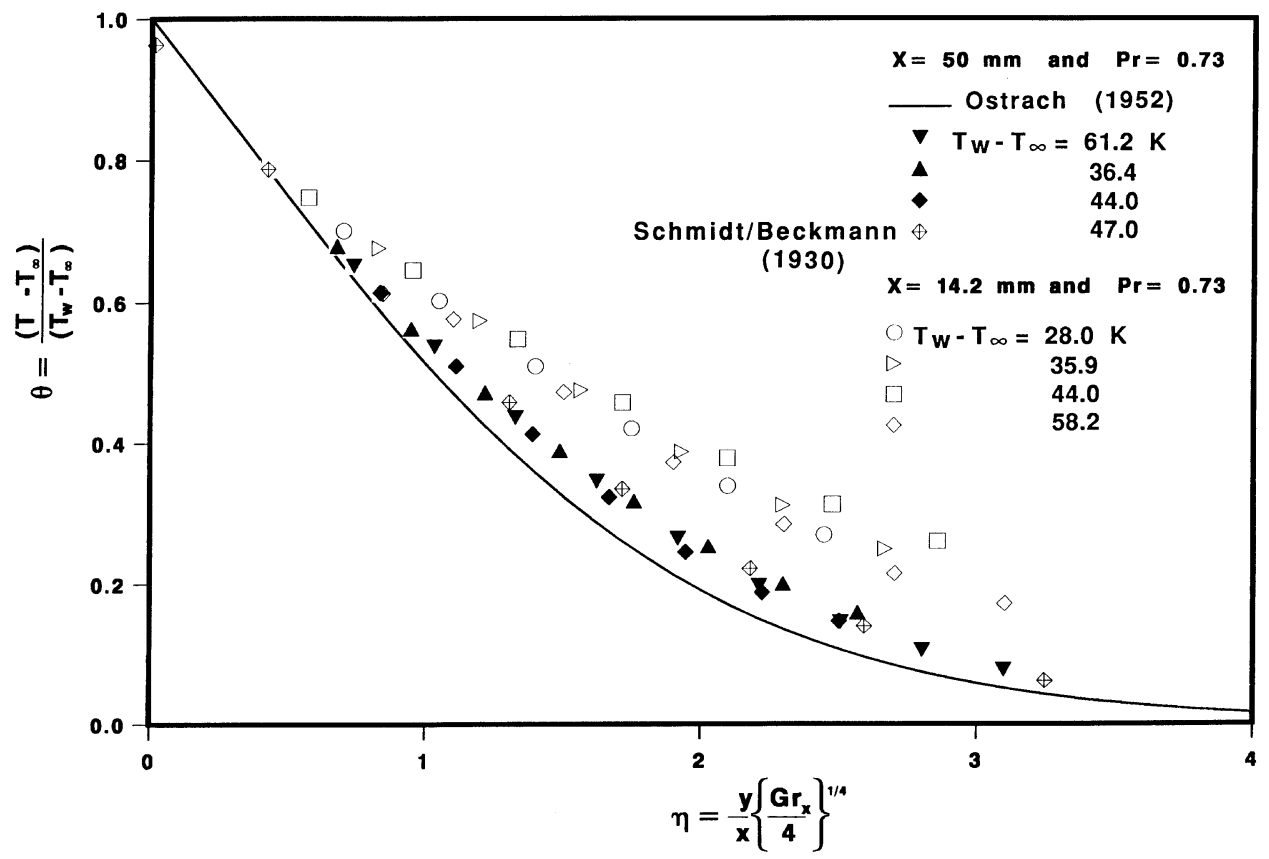

Fig. 7. Dimensionless temperature profiles as a function of dimensionless similarity variables measured at $x=14 \cdot 2$ and $x=50 \cdot 0 \mathrm{~mm}$ for different wall temperatures. ${ }^{24}$ 
The leading-edge effect is reduced and a better agreement is shown at $x=50.0 \mathrm{~mm}$ because it is away from the leading edge. A similar comparison of the speckle photography technique ${ }^{27}$ has also been made against a Wollaston prism interferometer and a Mach-Zehnder interferometer. All three methods showed good agreement in the region away from the leading edge. More recently, a combined optical system ${ }^{28}$ has been devised to simultaneously record a specklegram and a shearing interferogram using a pulsed ruby laser.

Figure 8 shows the laser speckle measurement results of the local Nusselt number versus Grashof number for vertical channel flows of different aspect ratios of $b / L$, with $b$ being the channel width and $L$ being the channel length. ${ }^{29}$ For opening ratios down to $0 \cdot 1$, each wall acts as independently developing separate boundary layers and the measured results show good agreement with the single-plate theory. ${ }^{25}$ For $b / L=0 \cdot 05$, however, the data start to deviate from the theory as the local Grashof number exceeds $5 \times 10^{4}$. The two thermal boundary layers start interfering with each other and the heat-transfer coefficient decreases because of thermal choking. ${ }^{30}$ Uniform temperature profiles prevail across the merged channel flow and no measurable fringes are detected. ${ }^{31}$ Given the condition in Fig. 8, the minimum channel opening for separate layer development is calculated to be $(b / L)_{\min }=0.0584$ when using the published experimental correlation. ${ }^{32}$

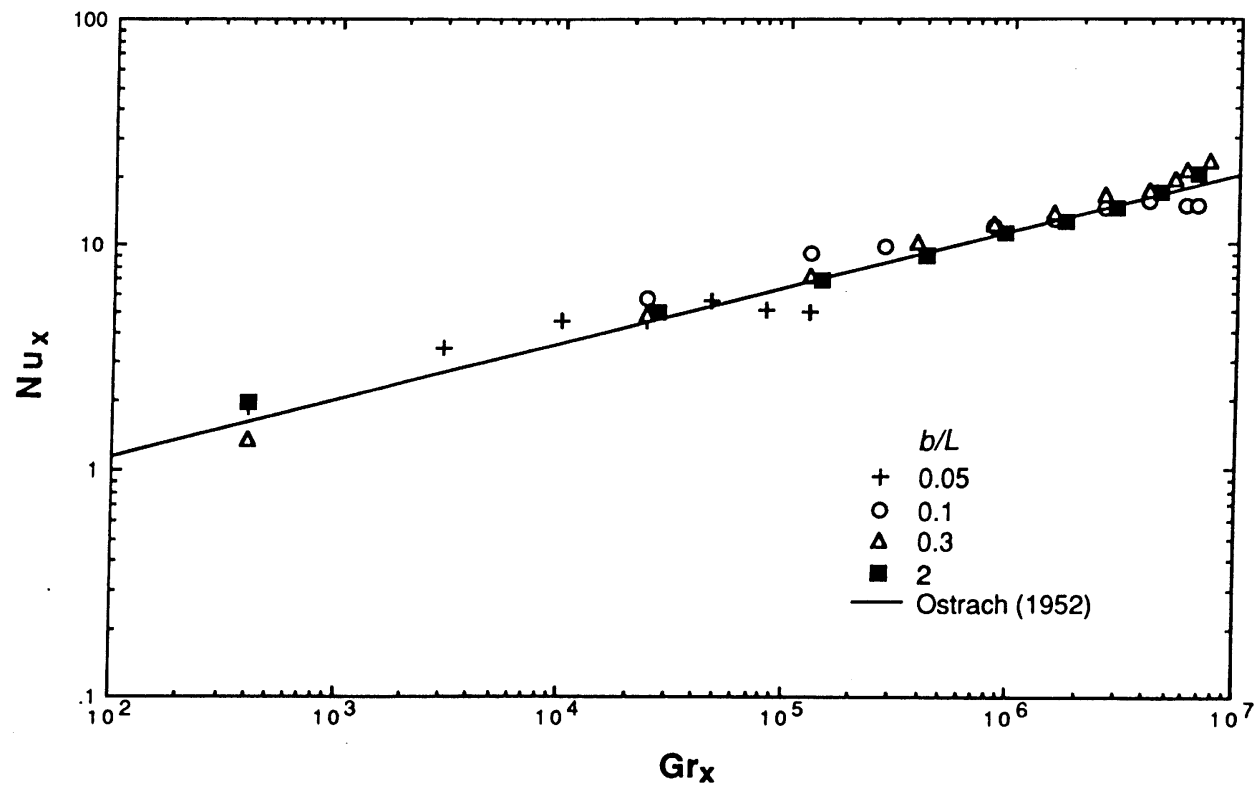

Fig. 8. Local Nusselt number versus local Grash of number for an isothermal vertical channel flow for different aspect ratios. ${ }^{29}$ 


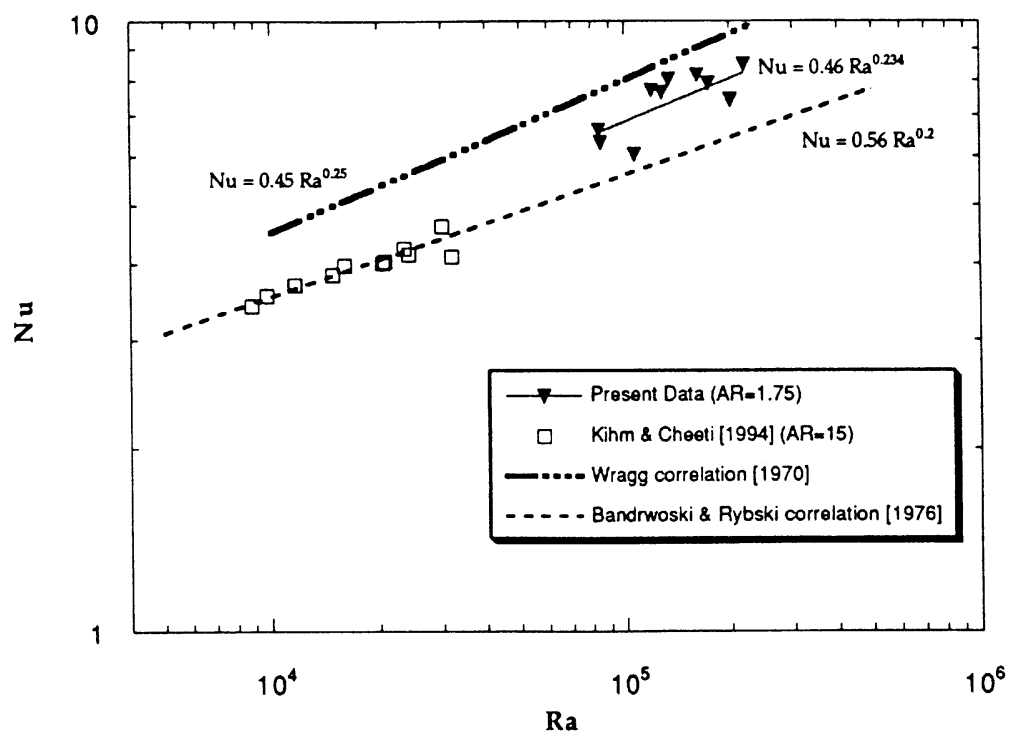

Fig. 9. Speckle results of average Nusselt number for a horizontal isothermal plate compared with other experimental and numerical results. ${ }^{35}$

The natural convection of an upward-facing isothermal plate has been widely studied, but is still under dispute, as it shows scattered results. ${ }^{33}$ Figure 9 shows the global Nusselt number data versus the Rayleigh number [a type of modified Grashof number, i.e., $\mathrm{Ra} \equiv \mathrm{Gr}_{b}(v / \alpha)(b / L)$ where $\alpha$ denotes thermal diffusivity] for two rectangular isothermal plates with aspect ratios of 1.75 and 15 , respectively. ${ }^{34}$ The speckle results fall in the band between the $1 / 4$ power law, derived from the experimental correlation using an electrochemical method for a square plate, ${ }^{35}$ and the $1 / 5$ power-law prediction, based on the similarity solution for an infinite plate using a mass-transfer analogy. ${ }^{36}$ The present results for the aspect ratios in between are well bounded by these two extreme conditions.

Natural convection-heat-transfer characteristics for vertically converging isothermal channel walls (Fig. 10) are studied using the laser speckle technique. ${ }^{29,37}$ Unlike a closed-triangular enclosure configuration with no openings, ${ }^{38,39}$ the natural convection boundary layers along the inclined heated surfaces will induce air flow from the lower openings. A sufficient top opening, $b$, will ventilate the induced flow [Fig. 10(a)], and effectively insulate the bottom plate (the ceiling in a building) from the heated inclined surfaces of length $L$ (the rooftop). On the other hand, when the top opening is too small [Fig. 10(b)], the two boundary layers merge inside and the convective air heat/mass transfer significantly decreases since a part of the induced/heated air recirculates within the enclosure (choked state). 


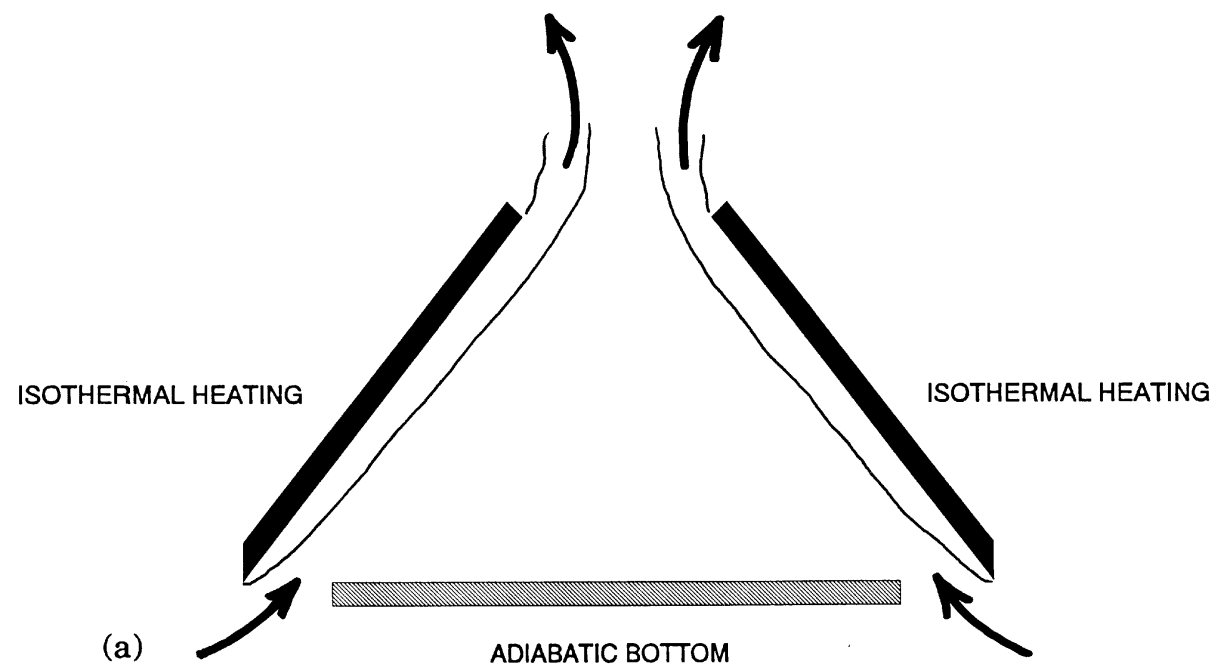

(b)

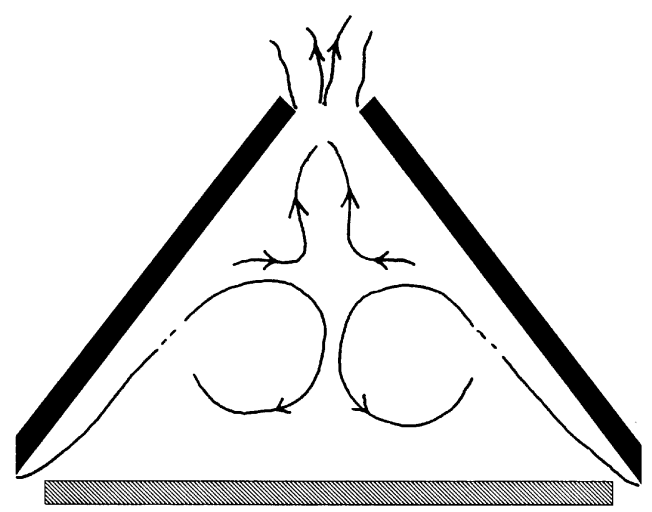

Fig. 10. Schematic illustrations of thermal boundary layers in a triangular enclosure: (a) complete ventilation with separate boundary layer development for $b / L>(b / L)_{\text {opt }_{3}}$ and (b) heated air recirculation with merging of two thermal layers for $b / L<(b / L)_{\text {opt }}{ }^{38}$

Figure 11 presents results of the local Nusselt number versus the Grashof number for a $30^{\circ}$ inclination from the vertical. The solid line represents the single inclined plate theory ${ }^{25}$ modified for the decreased gravitational effect due to the inclination. As the top opening ratio decrease below $b / L=0 \cdot 2$, the thermal boundary layers merge inside the channel and the heat transfer significantly decreases after merging, showing that $\mathrm{Nu}_{x}$ deviates from the theory.

Figure 12 shows the average Nusselt number versus the top opening ratio for the inclination angle $\gamma$ ranging from 0 to $60^{\circ}$ measured from the vertical. 


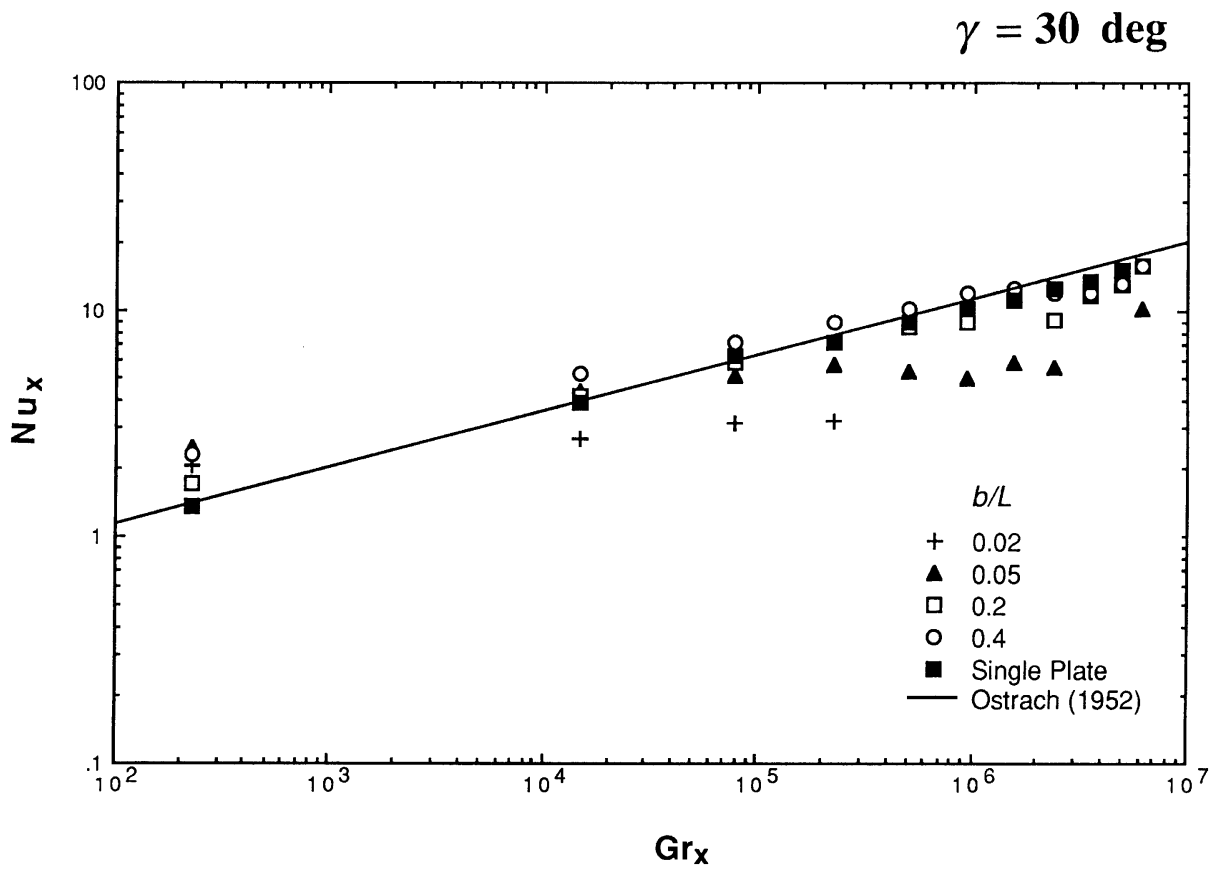

Fig. 11. Local Nusselt number verses local Grashof number for the converging flow with an inclination angle $\gamma=30^{\circ}$ measured from vertical. ${ }^{29}$

When $b / L$ is large, the average Nusselt number $\overline{\mathrm{Nu}}_{L}$ approaches the singleplate theory for all inclination angles. As $b / L$ decreases, a slight increase in $\overline{\mathrm{Nu}}_{L}$ is observed until the gradual ridge is reached. This maximum heat transfer occurs at an optimum opening ratio $(b / L)_{\text {opt }}$. The parallel channel $(\gamma=0)$ shows the smallest optimum opening ratio of $(b / L)_{\mathrm{opt}}=0 \cdot 07$, which is slightly larger compared to $(b / L)_{\min }=0.0584$ in a previous publication. ${ }^{32}$ The optimum opening ratio increases with increasing inclination angles of less steep rooftops. When the top opening decreases beyond the optimum, the average Nusselt number quickly decreases to less than the corresponding single-plate limit as the thermal choking prevails and lessens the heat transfer along the inclined surfaces.

\section{MEASUREMENT OF COMBUSTING FLAME TEMPERATURE PROFILES}

Refractive indices of most gas species gradually decrease with increasing gas temperature. ${ }^{40}$ The Gladstone-Dale relationship ${ }^{6}$ provides an expression for 


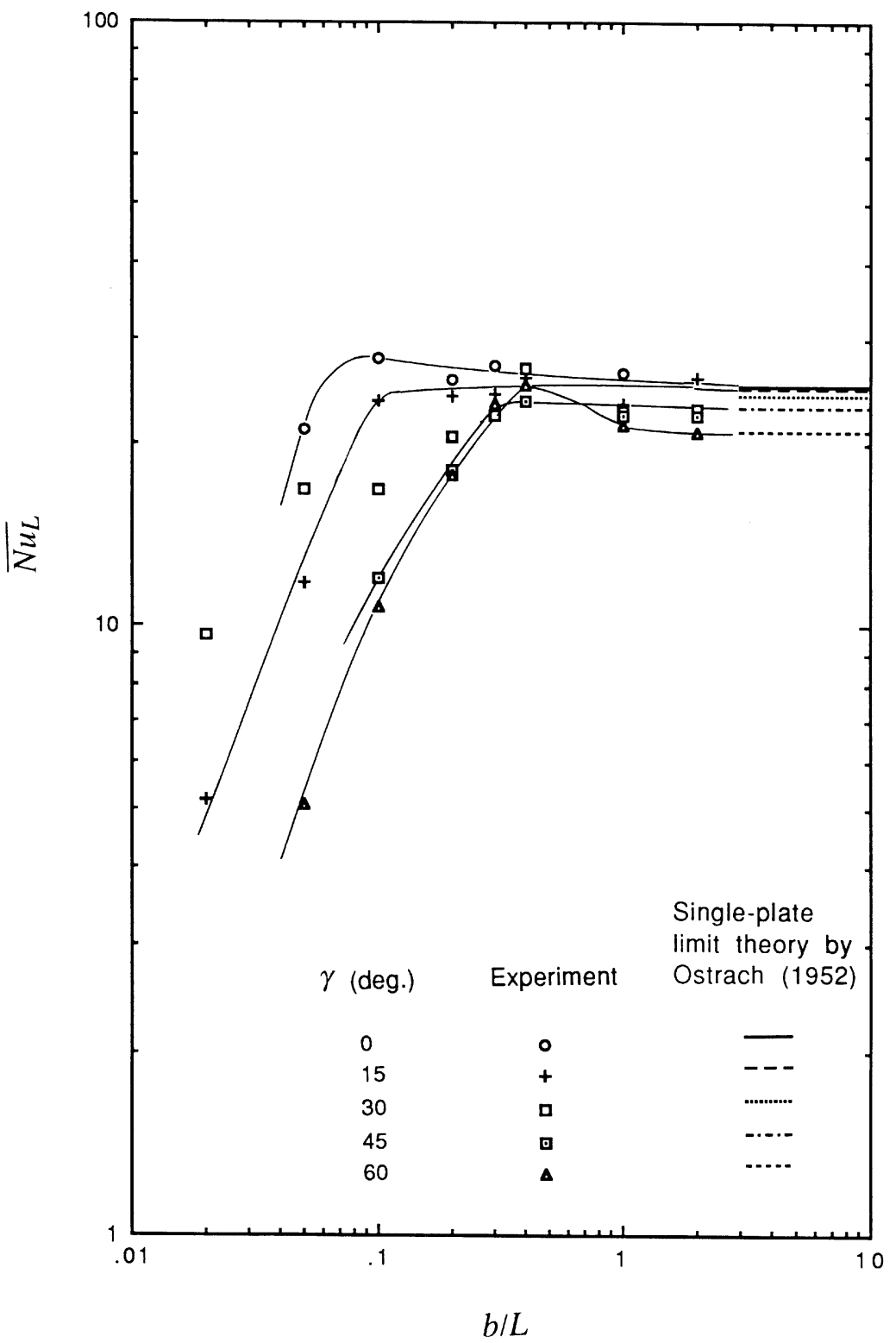

Fig. 12. Average Nusselt number based on the plate length versus the opening ratio for five different inclination angles: $\gamma=0$ (vertical channel), 15, 30, 45, and $60^{\circ}$; Grashof number $\left[g \beta \cos \gamma\left(T_{\mathrm{w}}-T_{\infty}\right) L^{3} / v^{2}\right]$ ranged from $3.58 \times 10^{6}\left(\gamma=60^{\circ}\right)$ to $7 \cdot 16 \times 10^{6}\left(\gamma=0^{\circ}\right) .{ }^{29}$

the index of refraction of air at 1 atm pressure as

$$
n(T)=1+K \rho=1+K \frac{P}{R T}=1+\frac{0 \cdot 07966}{T}
$$




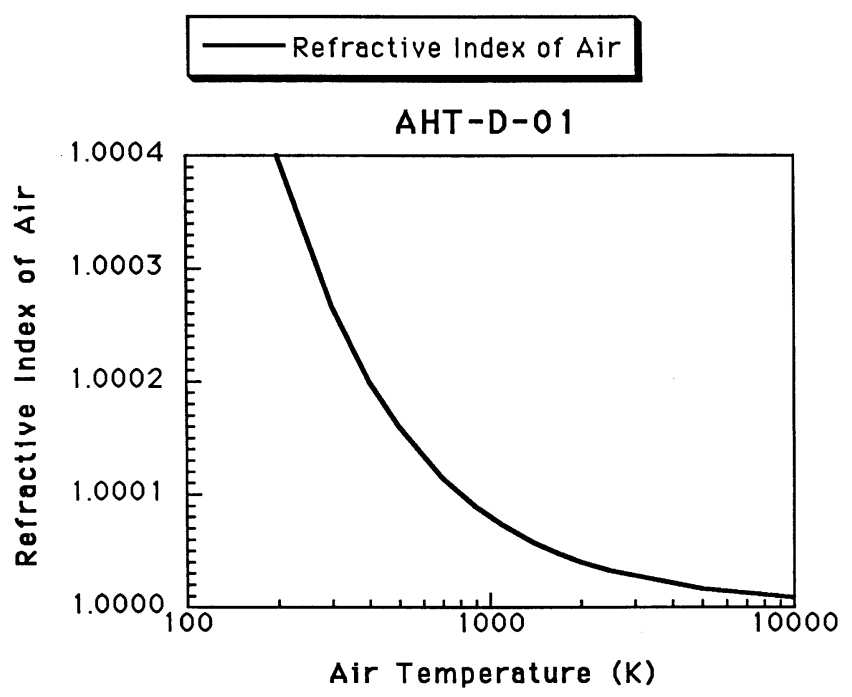

Fig. 13. Refractive index of air at $1 \mathrm{~atm}$ versus air temperature.

where $T$ is in degree Kelvin and the Gladstone-Dale constant $K=$ $0 \cdot 2257 \times 10^{-3} \mathrm{~m}^{3} / \mathrm{kg}$ at $\lambda=632.8 \mathrm{~nm}$ of a helium-neon laser. At a hightemperature environment, such as a combusting flame, the refractive index itself is low and also its gradient, $\mathrm{d} n / \mathrm{d} T=-0 \cdot 07966 / T^{2}$ [more accurately given in eqn (7)], approaches zero (Fig. 13). This implies that the change of refractive index per unit temperature significantly decreases at an elevated temperature. Thus, when applied for a flame temperature measurement, a ray refraction method, such as speckle photography, inherently reduces the measurement sensitivities.

Another difficulty in measuring flame temperature profiles arises from the fact that the specklegram is a line-of-sight projection recording containing integrated information of the cross-sectional field along the ray. Tomographic reconstruction of speckle data is necessary to recover the cross-sectional temperature distribution. This section discusses the axisymmetric test fields, which can be readily reconstructed by a mathematical inversion from a single projection, called Radon transformation. ${ }^{41}$ The following section discusses more general tomographic reconstruction problems for an asymmetric field that requires multiple projections.

The polar coordinate transformation of eqn (3a) for an axisymmetric flame field fixed at $z=z_{k}$ gives the measured deflection angle $\varepsilon(y)$ as an integral function of the radial index of refraction $n(r),{ }^{9}$

$$
\varepsilon(y)=2 y \int_{y}^{\infty} \frac{\partial}{\partial r}\left[\frac{n(r)}{n_{\mathrm{o}}}\right] \frac{\mathrm{d} r}{\sqrt{r^{2}-y^{2}}}
$$


where the $y-z$ imaging plane lies perpendicular to the optical axis $x$ and the $r$-coordinate lies in the $x-y$ plane (Fig. 5). It is assumed that the radial variation of refractive index dominates over its variation in the $z$-direction.

Equation (12) represents an integral transform of the Abel type, ${ }^{41}$ whose inverse Abel transform is given as

$$
\frac{n(r)}{n_{\mathrm{o}}}=1-\frac{1}{\pi} \int_{r}^{\infty} \frac{\varepsilon(y)}{\sqrt{y^{2}-r^{2}}} \mathrm{~d} y
$$

which is also called inverse Radon transform. Equation (13) reconstructs the refractive index distribution as a function of $r$ from the line-of-sight deflection angle data $\varepsilon(y)$.

The various combustion products can be approximated as air since their physical and optical properties are similar to those of air. ${ }^{42}$ Applying the Gladstone-Dale relation, $n=1+K \rho$, once for a reference point denoted by a subscript 'o' and for any arbitrary radial point ' $r$ ', and then combining the two equations with an ideal gas assumption gives ${ }^{9}$

$$
\frac{T(r)}{T_{\mathrm{o}}}=\left[\left(\frac{n(r)}{n_{\mathrm{o}}}-1\right)\left(\frac{R T_{\mathrm{o}}}{K p_{\mathrm{o}}}+1\right)+1\right]^{-1}
$$

where the pressure is assumed constant in the whole tested field.

An alternative conversion is possible by using the Lorentz-Lorentz relation, ${ }^{43} A \rho=\left(n^{2}-1\right) /\left(n^{2}+2\right)$, where $A$ and $\rho$ are the molar refractivity and molar density, respectively. An ideal gas assumption with an index of refraction $n \cong 1 \cdot 0$ gives an expression for local temperature as ${ }^{44}$

$$
\frac{T(r)}{T_{\mathrm{o}}}=\left[\left(\frac{n(r)}{n_{\mathrm{o}}}-1\right)\left(\frac{3 p_{\mathrm{o}} A+2 R T_{\mathrm{o}}}{3 p_{\mathrm{o}} A}\right)+1\right]^{-1}
$$

where a constant pressure $p_{\mathrm{o}}$ is assumed to prevail across the entire test field. To complete the temperature conversion, either by eqns (14) or (15), the reference temperature and index of refraction, $T_{\mathrm{o}}$ and $n_{\mathrm{o}}$, must be given at least at one point a priori.

The use of speckle interferometry ${ }^{45}$ can also determine the temperature field by analyzing the fringes appearing in a speckle interferogram. The analysis method is identical to that for the wavefront interferometric techniques such as Mach-Zehnder interferometry. ${ }^{46}$ For an axisymmetric field with $n \cong 1 \cdot 0$, the radial index of refraction is expressed by an integral equation of the line-of-sight fringe data $N_{\mathrm{f}}$, which is equivalent to the number of crossing fringes: ${ }^{47}$

$$
\frac{n(r)}{n_{\mathrm{o}}}=1-\frac{\lambda}{\pi} \int_{r}^{\infty} \frac{\left(\mathrm{d} N_{\mathrm{f}} / \mathrm{d} y\right)}{\sqrt{y^{2}-r^{2}}} \mathrm{~d} y
$$


Using the Lorentz-Lorentz equation ${ }^{43}$ and the assumption of an ideal gas, an equation for temperature conversion is given:

$$
\frac{T(r)}{T_{\mathrm{o}}}=\left[\frac{C}{T_{\mathrm{o}}}\left(\frac{n(r)}{n_{\mathrm{o}}}-1\right)\right]^{-1}
$$

where $C$ is an optical constant and is equal to $0.083928 \mathrm{~K}$ for a helium-neon laser. $T_{\mathrm{o}}$ is the temperature of a reference point where the refractive index is $n_{\mathrm{o}}$.

Because of their simplicity and symmetry, a candle flame is a popular example for testing various optical techniques. ${ }^{48-50}$ Figure 14 shows Young's fringe patterns constructed at different vertical $(z)$ and horizontal $(y)$ locations on the image plane of a diffusive candle flame. ${ }^{51}$ A narrower fringe pattern represents a larger refraction angle resulting from a higher-temperature gradient at one location, and vice versa for fringes with wider spacing at another location. Fringes with infinite spacing are observed along the centerline because of its symmetry, with no fringes at the outside edge because of a zero-temperature gradient. The vertically aligned fringes for all $y$-locations at $z=20 \mathrm{~mm}$ imply that the ray truly refracts within the plane with negligible
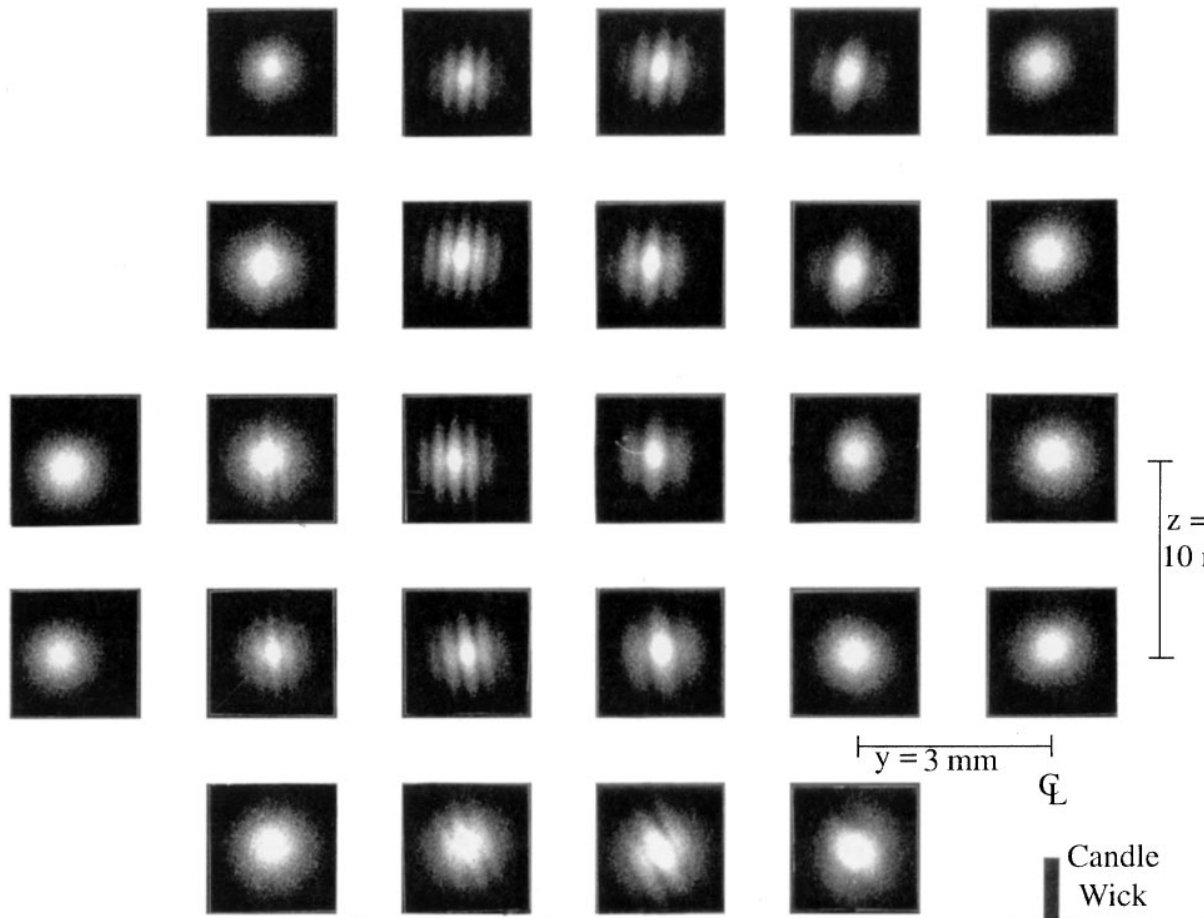

Fig. 14. Spatial distribution of Young's fringes generated from a laser specklegram recorded for an axisymmetric paraffin candle flame. ${ }^{51}$ 
refraction in the $z$-direction for that specific $z$-plane. The slightly tilted fringes at other $z$-planes show that the local temperature gradients have both radial and nonzero axial components.

Figure 15 shows the projected ray deflection angles evaluated at $z=20 \mathrm{~mm}$ from the fringe spacing measurement. The largest deflection angle is observed at approximately $y=9 \mathrm{~mm}$, corresponding to the location of the narrowest fringes shown in Fig. 14. The discrete data are curve-fitted in a piecewise manner: a linear increase near the center, a polynomial in the middle, and an exponential decay near the flame edge. Numerical integration of eqn (13) for the curve-fitted ray deflection $\varepsilon(y)$ determines the refractive index variation as a function of $r$ (Fig. 16). The reference value of refractive index $n_{\mathrm{o}}$ is taken for the ambient condition. The lowest index of refraction at the center of the candle flame corresponds to the highest centerline temperature.

Figure 17 shows the radial temperature distribution of a premixed propane Bunsen flame $50 \mathrm{~mm}$ downstream of the $8 \mathrm{~mm}$ diameter nozzle. ${ }^{52}$ The temperature field is converted from the measured and reconstructed index of refraction $n(r) / n_{\mathrm{o}}$ as shown in eqn (14). The solid curve shows a temperature profile determined using the specklegram data, with the centerline as a reference

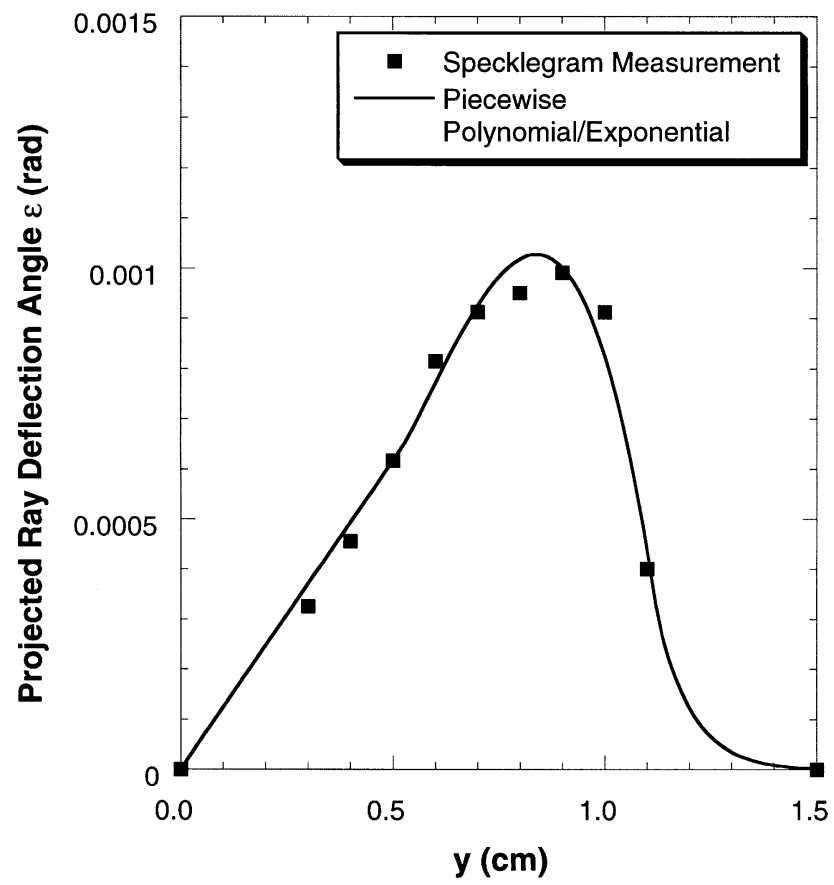

Fig. 15. Projected refraction angle measured directly from Young's fringes for an axisymmetric paraffin candle flame. ${ }^{51}$ 
Radial Distribution of Normalized Index of Refraction of Parafin Flame

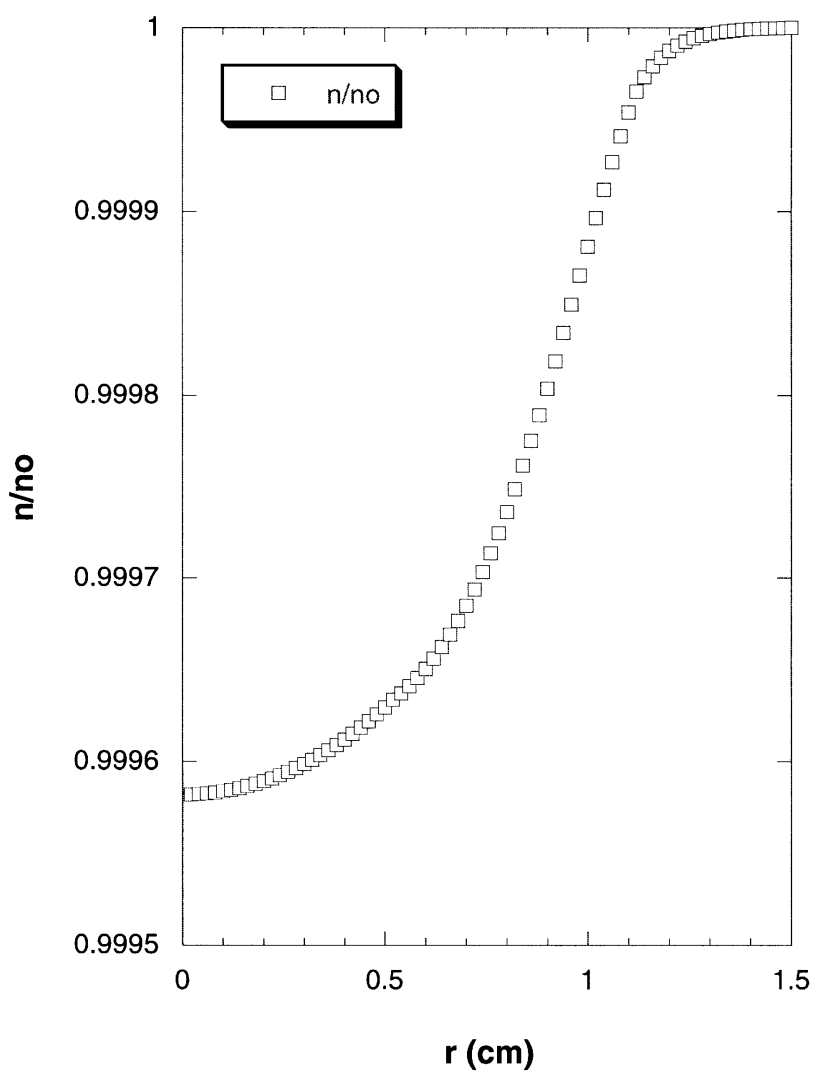

Fig. 16. Reconstructed radial distribution of refractive index of a paraffin candle flame. ${ }^{52}$

point, assuming both the gas constant for air and the Gladstone-Dale constant of air. The speckle data show fairly good consistency with the thermocouple (platinum-rhodium B-type) data, except near the flame boundary where the speckle data underpredicts the ambient temperature. The thermocouple data have not been attempted for any corrections for their radiation losses from the probe surface nor for the conduction heat losses through the probe stem. ${ }^{53}$ The probe corrections must know the emissivity of the probe surface, the time constant of the probe material, and the flow Reynolds number. The temperature correction for the heat losses could upshift the thermocouple data as much as $20 \%{ }^{54}$

The specific gas constant and the Gladstone-Dale constant must be estimated for the mixture of gases presented in the flame if the mass fractions of 


\section{Propane Flame Temperature Profiles}

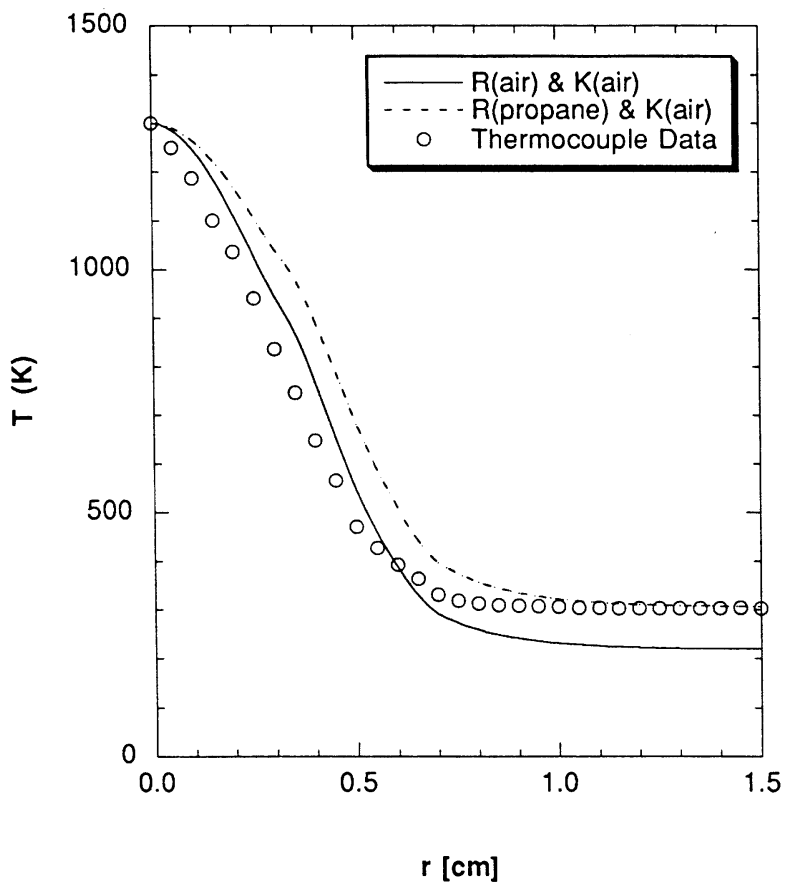

Fig. 17. Comparison of temperature profile obtained from reconstructed refractive index with thermocouple-measured temperature data for the axisymmetric propane Bunsen flame. ${ }^{52}$

the product gases are known or measured for the flame. ${ }^{55}$ The GladstoneDale constant of propane is approximated as that in air since the constant is a strong function of wavelength, but a weak function of gas species. ${ }^{42} \mathrm{How}$ ever, the gas constant $R$ varies rather significantly for gas species. The dashed curve represents a temperature profile calculated by assuming a gas constant for $100 \%$ propane while keeping the Gladstone-Dale constant for air. The discrepancy in the ambient region has been significantly diminished in this limit, whereas the midrange temperature profile shows slightly more deviations from the thermocouple data.

Other than the reduced sensitivity of refractive index at high-temperature medium, additional sources of uncertainties in measuring flame temperatures have been identified: (1) The thermocouple probe corrections would raise the thermocouple data near the center to a noticeable degree. This increase of the reference temperature $T_{\mathrm{o}}$ would also increase the speckle temperature data, particularly near the flame boundary. (2) The corrections for the nonradial angular refraction (occurring up to $20 \%$ at some locations ${ }^{9}$ ) would lower the 
specklegram temperature data and reduce the deviations from the thermocouple data in the midrange of the flame. (3) The discrepancy can also be attributed to the asymmetry of the flame that are expected to give additional $5-10 \%$ uncertainties.

\section{TOMOGRAPHIC RECONSTRUCTION OF SPECKLE PHOTOGRAPHY DATA}

Determining general asymmetric temperature or density field requires a set of specklegrams taken at different projection angles and these line-of-sight speckle data need to be reconstructed using an appropriate computer tomography (CT) algorithm. Most known algorithms belong to either a direct mathematical inversion from projections or a regressive optimization of the field that may be represented by a series expansion.

The former, typically called a Fourier convolution (FC), is based on the Fourier slice theorem. ${ }^{56}$ For the geometry shown for the speckle photographic tomography (Fig. 18), the ray deflection angle is given as

$$
\varepsilon(s, \theta)=K \int \frac{\partial \rho}{\partial s} \mathrm{~d} t
$$

where the integrand takes the density gradient in the lateral direction $s$ and the integral is taken along the ray direction $t$. By taking Fourier transforms on both sides of eqn (18) and after some mathematical rearrangement, ${ }^{57}$ the inverse Fourier transformation is derived to give the density distribution. The

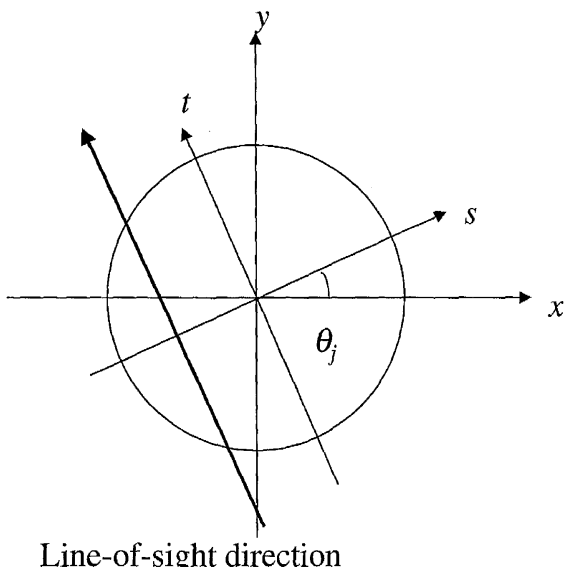

Fig. 18. Coordinate system of an arbitrary cross-section of test field. 
result is

$$
\hat{f}(x, y)=\rho(k \Delta x, n \Delta y)=(P \pi K)^{-1} \sum_{j=0}^{P-1} \sum_{m^{\prime}=-(M-1) / 2}^{(M-1) / 2} \varepsilon\left(m^{\prime} \Delta s, j \Delta \theta\right) q\left(m-m^{\prime}\right)
$$

where $\hat{f}$ is an object function to be reconstructed, $P$ denotes the number of projections, $K$ is the Gladstone-Dale constant, $k, n, j, m$ and $m^{\prime}$ are integers, and $\Delta x$ and $\Delta y$ are the grid sizes in $x$ and $y$ directions of the $M \times N$ discretized reconstruction plane.

The discretized filter function $q(m)$ is given as

$$
q(m)= \begin{cases}1 / m & \text { when } m \text { is odd } \\ 0 & \text { otherwise }\end{cases}
$$

where $m= \pm 1, \pm 2, \ldots, \pm(M-1) / 2$. The FC method is simple, very fast and accurate, but requires many equally angled projections for acceptable accuracy. Spatial and other constraints often limit the number of projections and restrict the view which allows partial projections. For such limited and restricted projections, the FC inversion method suffers severely from degraded accuracy.

The latter regressive optimization is represented by the algebraic reconstruction technique (ART), ${ }^{58}$ and usually needs fewer projections than the FC method. The ART is an iterative algorithm that reconstructs an object function $\hat{f}$ as a summation of basis functions $b_{i}$, i.e.,

$$
\hat{f(x, y)}=\sum_{i=1}^{M N} O_{i} b_{i}\left(x-x_{i}, y-y_{i}\right)
$$

where $O_{i}$ is the coefficient of the $i$ th basis function centered at $\left(x_{i}, y_{i}\right)$. A comparative study of the choice of basis functions suggests the use of the cubic B-spline. ${ }^{59}$ All reported ART results in this paper use the cubic B-spline basis function. Comparing the virtual projection of an intermediate guessed field, $\hat{f,}$ and the measured projection of the actual field, $f$, provides the feedback information, $I$, in terms of the discrepancies between the two projections:

$$
I_{p}=\left(\psi_{p}-\hat{\psi}_{p}\right)
$$

where $\psi_{p}$ and $\hat{\psi}_{p}$ represent the $p$ th measured and the $p$ th virtual projection, respectively. The ART uses the feedback information $I_{p}$ to iteratively update the object coefficient vector $O_{i}$.

For the case of an interferometric projection, the projection is directly expressed by the ray sum of the object field:

$$
\psi_{p}=\text { Const } \int_{t} f(x, y) \mathrm{d} t
$$


The ART updating scheme is given by combining eqns (21)-(23):

$$
O_{i}^{q+1}=O_{i}^{q}+W_{i} \frac{\sum_{p} I_{p}}{\text { Const } \cdot M N P}
$$

where $W_{i}$ is the weighting factor and $q$ denotes the $q$ th iteration.

The ART, represented by eqn (24), works fairly well in reconstructing algebraic projection of interferometry type ${ }^{15}$ where the projection data represent the ray integrals of the field property itself. However, for a nonalgebraic projection, such as speckle photography, the projection angle data represent the ray integral of the field gradient in the lateral direction [eqn (18)] and the ART updating scheme [eqn (24)] does not provide updating the field. The main reason is that the feedback $I_{p}$ contains the amount of discrepancies between the measured and virtual projections of the density gradient while the updating requires information regarding the discrepancy of the density field itself.

The ambiguity of ART occurring for nonalgebraic projections can be alleviated by modifying its regression procedure. ${ }^{60}$ First, the nonalgebraic speckle data of ray deflection angles are integrated on the image plane to convert into the algebraic fringe number data of interferometry. A phase object creates the path length difference between the reference beam and the test beam of interferometry. The resulting fringes are then shifted with respect to the undisturbed fringes by a fringe shift number $\alpha$, i.e.,

$$
\alpha=\frac{1}{\lambda} \int_{-\infty}^{\infty}\left(n-n_{\text {ref }}\right) \mathrm{d} t=\frac{K}{\lambda} \int_{-\infty}^{\infty}\left(\rho-\rho_{\text {ref }}\right) \mathrm{d} t
$$

Comparing the fringe shift number with the ray deflection angle [eqn (18)] gives

$$
\lambda \frac{\partial \alpha}{\partial s}=K \int \frac{\partial \rho}{\partial s} \mathrm{~d} t=\varepsilon
$$

Integrating eqn (26) along the horizontal direction $s$ on the projection plane gives

$$
\alpha=\frac{1}{\lambda} \int \varepsilon \mathrm{d} s
$$

Equation (27) implies that an integral of the ray deflection angle recorded on the specklegram, $\varepsilon$, along $s$, gives the corresponding interferometric fringe shift number $\alpha$. Now, the ART reconstructs the field from the converted interferometric data that are algebraic. The modified ART works fairly well for speckle photography, but the numerical integral of discrete speckle data may be subjected to an additional computational errors. 
The modified ART is tested for use with the speckle photography and considers a smooth and asymmetric computer-synthesized phantom density field [Fig. 19(a)]: ${ }^{61}$

$f(x, y)$

$$
=\left\{\begin{array}{ll}
\rho(x, y)=\frac{\left[1-\cos \left\{2 \pi(x+0 \cdot 5)^{4 / 5}\right\}\right]\left[1-\cos \left\{2 \pi(y+0 \cdot 5)^{2 / 3}\right\}\right]}{4}, & |x, y|<0 \cdot 5 \\
0 & \text { otherwise }
\end{array} .\right.
$$

The ART reconstruction results [Figs 19(d) and (e)] are compared with the FC results [Figs 19(b) and (c)]. The comparison is made under two different equally angled projections of five and three. Each projection consists of 45 rays giving 45 beam deflection readings. The $\mathrm{FC}$ calculations consider $15 \times 15$ $(M=N=15)$ discrete nodal points (square pixels) and the ART calculations consider $15 \times 15$ cubic-B spline type basis functions.

The FC reconstruction suffers more severely from limited projections. Under five projections, the ART reconstructs the field with fairly good accuracy, but the FC reconstruction shows a high level of noise. Under three projections, which may be regarded as the minimally required number of projections to perceive an asymmetric object no matter how simple the shape takes, the ART is still able to reconstruct the field with acceptable accuracy. The FC result, however, shows contours distorted beyond recognition except for a glimpse of an approximate location of the peak.

The degraded accuracy of the FC under limited projections is more pronounced for the case of a double-peak phantom (Fig. 20a): ${ }^{61}$

$$
\begin{aligned}
f(x, y)= & 1 \cdot 09\left[0 \cdot 3 \cos (x, y)+0 \cdot 8\left(\mathrm{e}^{\left\{-\left[9\left(x-x_{1}\right)\right]^{2}-\left[6\left(y-y_{1}\right)\right]^{2}\right\}}\right.\right. \\
& \left.\left.+\mathrm{e}^{\left\{-\left[8\left(x-x_{2}\right)\right]^{2}-\left[30\left(y-y_{2}\right)\right]^{2}\right\}}\right)\right]
\end{aligned}
$$

Reconstruction calculations are performed for $25 \times 25$ nodal points $(\mathrm{FC})$ or $25 \times 25$ cubic-B spline basis functions $($ ART) under five projections $(M=$ $N=25, P=5$ ), with each projection consisting of 63 rays. The ART results are still marginally acceptable for both surface and contour plots (Fig. 20b) while the FC results are very noisy and give hardly any clue of the phantom (Fig. 20c). Although not shown, both FC and ART results under three projections are beyond recognition.

A modification is made to tomographically reconstruct nonalgebraic speckle photography projections using ART. An integration of the ray 

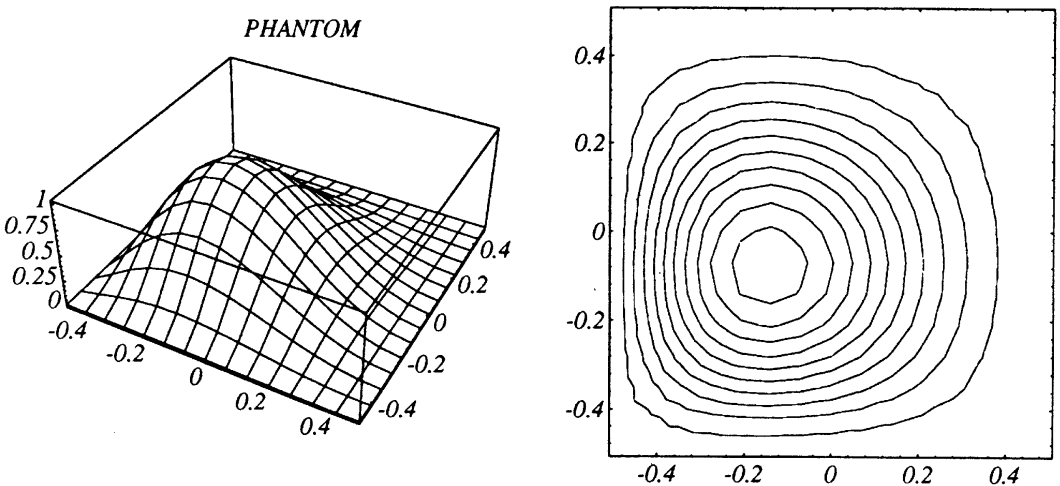

(a)

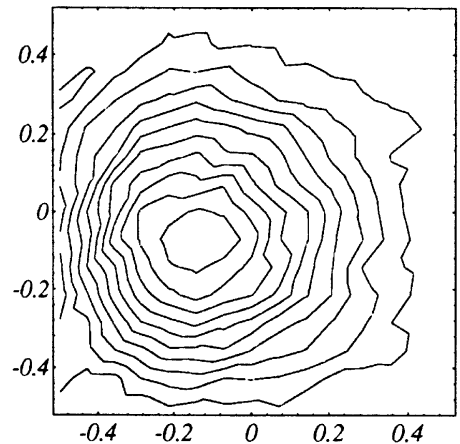

(b)

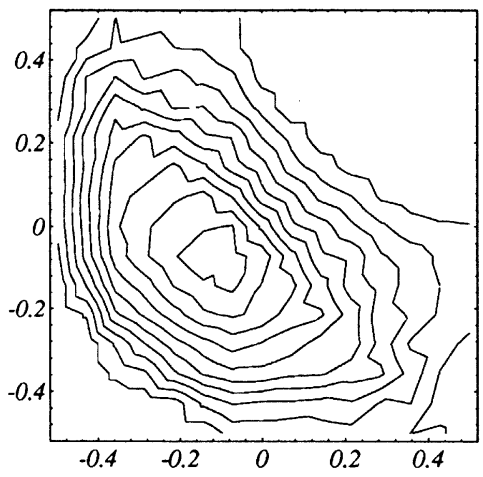

(c)

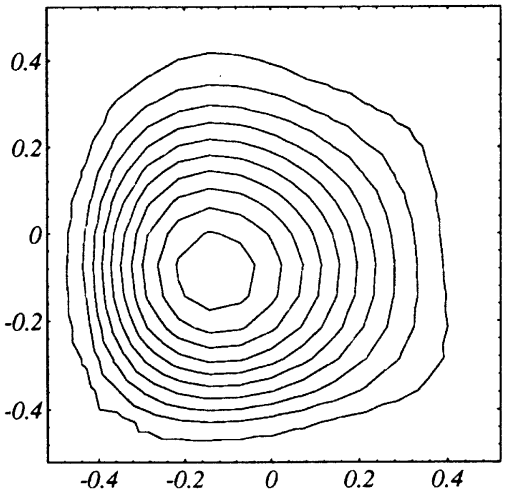

(d)

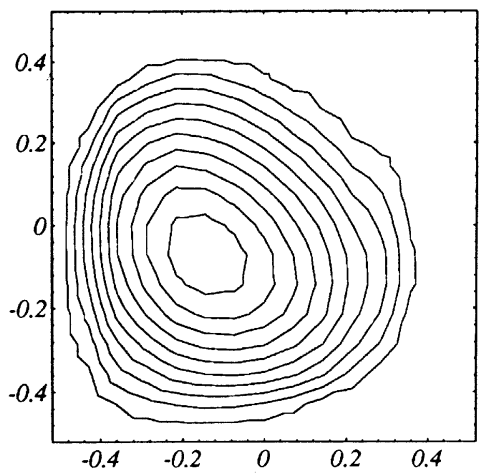

(e)

Fig. 19. Asymmetric single-peak phantom (a), and its reconstruction using FC (b, c) and ART $(d, e)$ under five $(b, d)$ and three $(c, e)$ projections. 

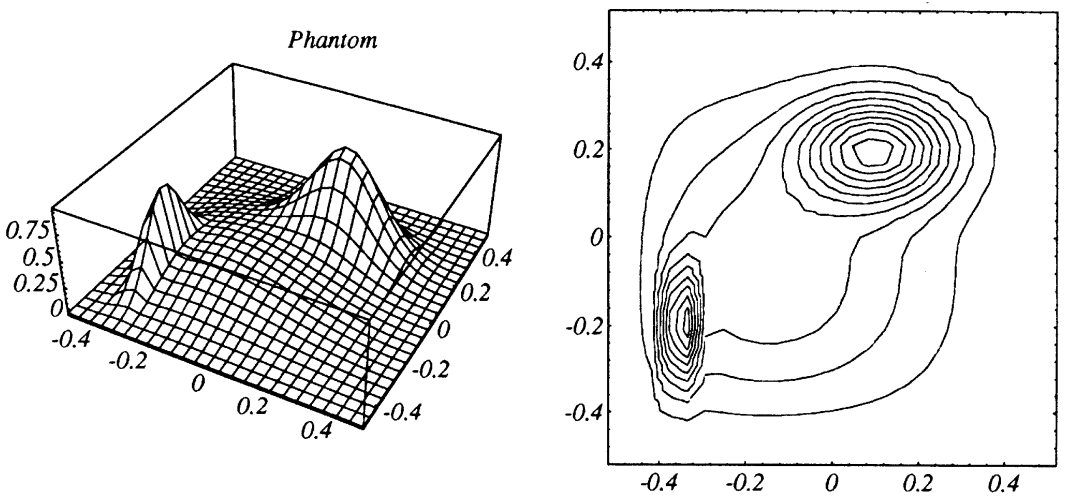

(a)
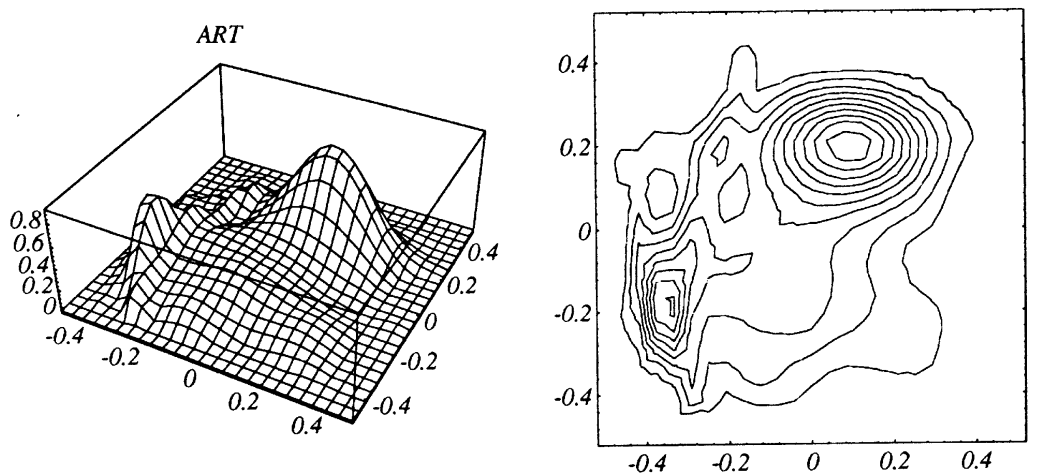

(b)
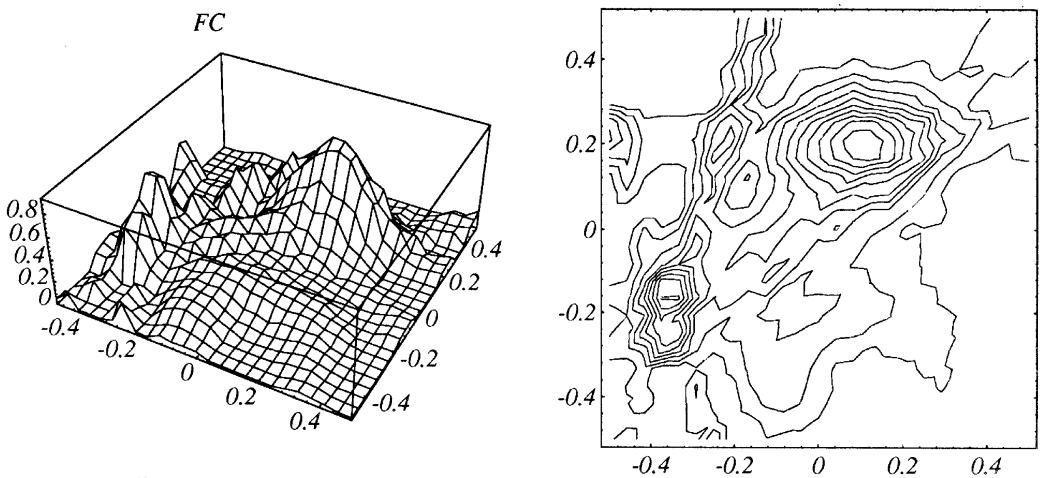

(c)

Fig. 20. Asymmetric twin-peak phantom (a), and its reconstruction using ART (b), and FC (c) under five projections. 
deflection angles on the projected plane converts into the interferometric and algebraic fringe crossing number data. The modified ART method shows noticeably less suffering from limited projections in comparison with the conventional FC method.

\section{CONCLUDING REMARKS}

The operating principle of laser speckle photography is reviewed and its applications to selected thermal flow problems are discussed. The technique measures natural convection coefficients for various heat-transfer problems: a single vertical heated plate and a vertical channel as reference geometry that have been used for comparison with other techniques, upward-facing isothermal plates for the case under dispute, and converging isothermal channel walls with different converging angles as a representation of a heat transfer between the rooftop and ceiling. Laser speckle photography nonintrusively and conveniently measures the heat-transfer coefficients with high resolution and no cumbersome corrections or estimation are necessary.

High-temperature applications of laser speckle photography inherently suffer from measurement uncertainties because of the decreasing temperature dependence of refractive index with increasing temperature. In addition, tomographic reconstruction of the line-of-sight speckle data is necessary to measure the radial temperature profiles for combusting flames. The results obtained for laminar axisymmetric premixed flames show some deviations compared with the uncorrected thermocouple data. Accurate conversion from the raw data of refractive index to temperature requires known mole fractions of combustion products and the Gladstone-Dale constant must be appropriately evaluated. Other aspects for improvement include the corrections for axial refraction and incorporation of the flame asymmetry.

Computer-synthesized phantoms of asymmetric single- and double-peak density fields have examined the efficacy of the laser speckle tomography. For limited projections, which is not unusual for many practical cases, FC reconstructs the fields poorly because of insufficient information for the direct mathematical conversion. The modified algebraic reconstruction technique (ART) enables the reconstruction of nonalgebraic speckle projection data. The projected-beam-deflection data are integrated to convert into the algebraic fringe number data of interferometry. The modified ART gives a noticeably better reconstruction under equally limited and restricted projections compared with the FC conversion. Therefore, the modified ART is recommended to use for tomographic reconstruction of the laser speckle photographic projection in lieu of Fourier convolution. 


\section{REFERENCES}

1. Françon, M., Laser Speckle and Applications in Optics. Academic Press, New York, 1979.

2. Adrian, R. J., Scattering particle characteristics and their effect on pulsed laser measurements of fluid flow: speckle velocimetry vs particle image velocimetry. Appl. Opt., 23 (1984) 1690-1.

3. Debrus, S., Françon, M., Grover, G. P., May, M., \& Roblin, M. L., Ground glass differential interferometer. Appl. Opt. 11 (1972) 853-7.

4. Köpf, U., Application of speckling for measuring the deflection of laser light by phase objects. Opt. Commun., 5 (1972) 347-50.

5. Debrus, S. \& Grover, C. P., Correlation of light beams scattered at different angles by a ground glass. Opt. Commun., 3 (1971) 340-2.

6. Merzkirch, W., Flow Visualization, 2nd edn. Academic Press, New York, 1987.

7. van de Hulst, H. C., Light Scattering by Small Particles. Dover, New York, 1957.

8. Li, Q. B. \& Chiang, F. P., Three-dimensional dimension of laser speckle. Appl. Opt., 31 (1992) 6287-91.

9. Walsh, T. E., A comparative study of laser speckle photography and laser interferometry for optical tomography. PhD Dissertation, Department of Mechanical Engineering, Texas A\&M University, College Station, TX, 1996.

10. Kihm, K. D., Image blurring of test section boundary in a specklegram technique for temperature gradient measurements. Appl. Opt., 31 (1992) 5907-10.

11. Archbold, E., Burch, J. M. \& Ennos, A. E., Recording of inplane surface displacement by double-exposure speckle photography. Opt. Acta., 17 (1970) 883-93.

12. Hecht, E., Optics. Addison-Wesley, New York, 1974.

13. Weyl, F. J., Analysis of optical methods. In Physical Measurements in Gas Dynamics and Combustion, ed. R. W. Ladenburg. Princeton University Press, Princeton, 1954, pp. 3-25.

14. Mewes, D., Advances in optical methods for visualizing and measuring transport phenomena. Proc. 2nd Int. Symp. Transport Phenomena, Beijing, China, 1992, pp. $1-16$.

15. Vest, C. M., Holographic Interferometry. Wiley, New York, 1979.

16. Partington, J. R., An Advanced Treatise on Physical Chemistry, Physico-Chemical Optics, Vol. IV. Longmans Green, London, 1953.

17. Kihm, K. D., On the application of a laser specklegram technique for convective heat transfer problems. Proc. 6th Int. Symp. Transport Phenomena in Thermal Engineering, Seoul, Korea, 1993, pp. 1211-7.

18. Holman, J. P., Heat Transfer. McGraw-Hill, New York, 1963.

19. Elenbaas, W., Heat dissipation of parallel plates by free convection. Physica, 9 (1942) 1-28.

20. Eckert, E. R. \& Carlson, O., Natural convection in an air layer enclosed between two vertical plates with different temperatures. Int. J. Heat Mass Transfer, 2 (1961) 106-20.

21. Levy, E. K., Optimum plate spacing for laminar natural convection heat transfer from parallel vertical isothermal flat plates. J. Heat Transfer, 93 (1972) 463-5.

22. Wirtz, R. A. \& Stutzman, R. J., Experiments on free convection between vertical plates with symmetric heating. J. Heat Transfer, 104 (1982) 501-7. 
23. Anand, N. K., Kim, S. H., \& Fletcher, L. S., The effect of plate spacing on free convection between heated parallel plates. Proc. ASME Winter Annual Meeting, Vol. HTD-153, 1991, pp. 81-87.

24. Kastell, D., Kihm, K. D. \& Fletcher, L. S., Study of laminar thermal boundary layers occurring around the leading edge of a vertical isothermal wall using a specklegram technique. Exp. Fluids, 13 (1992) 249-56.

25. Ostrach, S., An analysis of laminar free-convection flow and heat transfer about a flat plate parallel to the direction of the generating body force. NACA Technical Note No. 2635, 1952.

26. Schmidt, E. \& Beckmann, W., Das Temperatur und Geschwindigkeitsfeld von einer Warme abgebenden senkrechten Platte bei naturlicher Konvektion. Tech. Mech. Thermodynam., 1 (1930) 341-9 and 391-406.

27. Wernekinck, U. \& Merzkirch, W., Measurement of natural convection by speckle photography. In Heat Transfer 1986, eds C. L. Tien et al. Hemisphere, Washington, 1986, pp. 531-35.

28. Shu, J. Z. \& Li, J. Y., A laser Schlieren-speckle interferometry system for measurement of phase objects. J. Flow Visualization Image Processing, 1 (1993) 63-8.

29. Kihm, K. D., Kim, J. H. \& Fletcher, L. S., Investigation of natural convection heat transfer in converging channel flows using a specklegram technique. J. Heat Transfer, 115 (1993) 140-8.

30. Kihm, K. D., Kim, J. H. \& Fletcher, L. S., Onset of flow reversal and penetration length of natural convective flow between isothermal vertical walls. J. Heat Transfer, 117 (1995) 776-9.

31. Guo, Z. -Y., Song, Y. -Z. \& Zhao, X. -W., Experimental investigation on natural convection in channel by laser speckle photography. Exp. Thermal Fluid Sci., 4 (1991) 594-600.

32. Bar-Cohen, A. \& Rohsenow, W. M., Thermally optimum spacing of vertical, natural convection cooled, parallel plates. J. Heat Transfer, 106 (1984) 116-23.

33. Goldstein, R. J. \& Lau, K. S., Laminar natural convection from a horizontal plate and influence of plate-edge extensions. J. Fluid Mech., 129 (1983) 55-75.

34. Kihm, K. D. \& Cheeti, S. K. R., Study of thermal flows from two-dimensional, upward-facing isothermal surfaces using a laser speckle photography technique. Exp. Fluids 17 (1994) 246-52.

35. Wragg, A. A. \& Loomba, R. P., Free convection flow patterns at horizontal surfaces with ionic mass transfer. Int. J. Heat Mass Transfer, 13 (1970) 439-42.

36. Bandrowski, J. \& Rybski, W., Free convenction mass transfer from horizontal plates. Int. J. Heat Mass Transfer, 19 (1976) 827-38.

37. Kihm, K. D., Kim, J. H. \& Fletcher, L. S., Effects of partial openings on natural convection heat transfer in triangular enclosures. Proc. ASME-Winter Annual Meeting, Vol. HTD-117, 1991, pp. 51-7.

38. Flack, R. D., Konopnicki, T. T. \& Rooke, J. H., The measurement of natural convective heat transfer in triangular enclosures. J. Heat transfer, 101 (1979) 648-54.

39. Flack, R. D., The experimental measurement of natural convection heat transfer in triangular enclosures heated or cooled from below. J. Heat Transfer, 102 (1980) $770-2$.

40. Chemical Rubber Company, CRC Handbook of Chemistry and Physics, 69th edn. CRC Press, Boca Raton, 1988. 
41. Deans, S. R., The Radon Transform and Some of its Applications. Wiley, New York, 1983.

42. Gardiner, W. C., Hidaka, Y. \& Tanzawa, T., Refractivity of combustion gases. Combust. Flame, 40 (1981) 213-9.

43. Born, M. \& Wolf, E., Principles of Optics. Pergamon Press, Oxford, 1980.

44. Farrell, P. V. \& Hofeldt, D. L., Temperature measurement in gases using speckle photography. Appl. Opt. 23 (1984) 1055-9.

45. Goodman, J. W., Speckle interferometry, In Laser Speckle and Related Phenomena, ed. J. C. Dainty. Springer, Berlin, Germany, 1975.

46. Michael, Y. C., Three-dimensional temperature reconstruction using Mach-Zehnder interferometric tomography. PhD Dissertation, Dept. Aerospace and Mechanical Engineering, University of Notre Dame, South Bend, 1991.

47. Verhoeven, D. D. \& Farrell, P. V., Speckle interferometry in transparent media. Appl. Opt., 25 (1986) 903-6.

48. Barakat, N., El-Ghandoor, H., Hamed, A. M. \& Diab, S., Refractive index profiling across a candle flame using speckle techniques. Exp. Fluids, 16 (1993) $42-5$.

49. Fomin, N. A., Multidirectional speckle photography of density fields in gasdynamics flows. Inzhenerno-Fizicheskii Z., 56 (1989) 540-7 (translated).

50. Blinkov, G. N., Fomin, N. A., Rolin, M. N., Soloukhin, R. I., Vitkin, D. E. \& Yadrevskaya, N. L., Speckle tomography of a gas flame. Exp. Fluids, 8 (1989) 72-6.

51. Walsh, T. E. \& Kihm, K. D., Tomographic deconvolution of laser speckle photography for axisymmetric flame temperature measurement. J. Flow Visualization Image Processing, 2 (1995) 299-310.

52. Walsh, T. E. \& Kihm, K. D., Tomographic deconvolution of laser speckle photography applied for flame temperature measurement. Proc. 7th Int. Symp. Flow Visualization, Seattle, 1995, pp 898-903.

53. ASTM, Manual on the Use of Thermocouples in Temperature Measurement, 4th edn. ASTM Press, Philadelphia, 1993.

54. Moffat, R. J., The gradient approach to thermocouple circuitry. Measurement Control Sci. Ind., 3 (1962) 33-7.

55. Weinberg, F. J., Optics of flames. Butterworths, Washington, 1963.

56. Kak, A. C. \& Slaney, M., Principles of Computerized Tomographic Imaging. IEEE Press, New York, 1987.

57. Liu, T. C., Merzkirch, W. \& Oberste-Lehn, K., Optical tomography applied to speckle photographic measurement of asymmetric flows with variable density. Exp. Fluids, 7 (1989) 157-63.

58. Gordon, R., A Tutorial on ART. IEEE Trans. Nucl. Sci., 21 (1974) 78.

59. Hanson, K. M. \& Wecksung, G. W., Local basis function approach to computed tomography. Appl. Opt., 24 (1985) 4028-39.

60. Ko, H. S., Lyons, D. P. \& Kihm, K. D., A comparative study of algebraic reconstruction technique (ART) and genetic algorithm (GA) for beam deflection tomography, ASME-FED 97 Summer Meeting, Paper No. FEDSM97-3104, Vancouver, Canada, June 1997.

61. Verhoeven, D., Limited-data computed tomography algorithms for the physical sciences. Appl. Opt., 32 (1993) 3736-54. 